\title{
Smart Mobile Gateway: Technical Challenges for Converged Wireless Sensor Networks and Mobile Cellular Networks
}

\author{
Lianhai Shan ${ }^{1,2}$, Weidong Fang ${ }^{2}$, Yunzhou Qiu ${ }^{2}$, Wei $\mathrm{He}^{2}$ and Yanzan Sun ${ }^{3}$ \\ ${ }^{1}$ Shanghai Research Center for Wireless Communications (WiCO), Shanghai, \\ China, Shanghai IoT Co. Ltd. \\ ${ }^{2}$ Shanghai Institute of Microsystem and Information echnology, Chinese \\ Academy of Sciences, Shanghai, China \\ ${ }^{3}$ Key laboratory of specialty fiber optics and optical access networks, Shanghai \\ University, Shanghai, China \\ Corresponding author: weidong.fang@mail.sim.ac.cn
}

\begin{abstract}
With the continued requirement of internet of things (IoT) development, many researchers focused on developing access and routing algorithms optimization of wireless sensor networks (WSNs) for communications quality guarantee, but less research has focused on the optimization of WSNs based on the mobile cellular networks (MCNs) assist. In this paper, we propose a convergence architecture of WSN and MCN for IoT applications, especially on the smart user equipment (UE) acting as a mobile gateway for WSN data transmission. And then, we investigate system application requirement for IoT. Finally, we discuss the key technical challenges and research points based on an analysis of smart gateway.
\end{abstract}

Keywords: Internet of Things; Smart mobile gateway; WSN; MCN; Converged networks

\section{Introduction}

With the continued development of information society, wireless sensor networks (WSNs) generated an increasing interest in industrial and research perspectives. WSNs have played a vital role in our daily lives, i.e. e-Health care, environment monitoring, industrial metering, surveillance systems et al. WSN can be generally described as a network of nodes that cooperatively sense and provide interaction between persons or computers and the surrounding environment [1]. The standardization process of WSNs is very active in recent years. An important outcome is represented by IEEE $802.11 \mathrm{x}$, 802.15.1, 802.15.3 and 802.15.4 et al, which is a short-range communication system to provide applications with relaxed throughput and service requirements in wireless personal area Networks (WPAN), and others technologies such as ultra wide band (UWB), Bluetooth, Zigbee et al.

There have been many researches in improving WSN system network structures (i.e. Flat or Hierarchical) [2]. In the hierarchical architecture, grouping sensor nodes into cluster has been widely pursued in order to achieve the WSN scalability objective [3]. The cluster head $(\mathrm{CH})$ is responsible for not only the general request but also receiving the sensed data of other sensor nodes in the same cluster and routing (transmitting) these data to the gateway [4]. Thus, the CHs have higher energy cost because all of the transmitting data packet will pass through them and be sent to the gateway. And the end-to-end transmission delay may become large, especially for the specific WSN areas based on belt-shaped topology or linear topology. In the flat architecture, there are energy and service aware multi-hop routing protocols. However, some technical issues 
must be considered with the increasing hop number among the sensor nodes [5] [6]. In current researches, some controllably mobile infrastructure for WSN is proposed in reference [7-8]. The WSN node will collect the data and transmit to the base station (BS) through mobile sink. This can greatly decrease the traditional transmission energy cost and transmission delay [9]. However, the serving mobile sink will not tell each WSN node about its leaving when it is leaving its responsible area, which will cause extra energy cast for communications [10]. And so, the mobile gateway is appearing in order to satisfy the requirement of WSN.

In the converged WSN-MCN system, WSN is described as a network of sensing nodes and can interact with the surrounding monitored things [11], which can be flexibly deployed to detect different types of data. In a WSN networks, the smart mobile user equipment (UE) gateway moves into the coverage area of the sensor nodes, they broadcast beacon packets to these nodes, and provide the backhaul access to these WSN nodes. MCN can send the detected WSN data directly to a central data center. MCN and WSN convergence is indispensable for supporting application of internet of things (IoT) [12-13], because MCN has the advantages of large coverage, powerful mobile terminals and mobile terminal can act as gateway to control the WSN nodes. The convergence of MCN and WSN can benefit each other: (I) For WSN, the MCN and mobile gateway can provide optimization to prolong the WSN life time, provide quality of service (QoS) for WSN; (II) For MCN, WSN can extend the intelligent application range of $\mathrm{MCN}$, i.e. WSN can provide real-time measurement results to $\mathrm{MCN}$ users [14]. The convergent interactive control and joint optimization technologies of MCN and WSN is based on the smart mobile gateway's application. Hence, the smart mobile gateway needs to be researched and developed for IoT application.

In the remainder of paper, we describe the converged WSN-MCN architecture and requirements for IoT in Section II. In Section III, we introduce the joint optimization of MCN and WSN for QoS guarantee. Moreover, the comprehensive system research issues and technical challenges are given in Section IV. Finally, we conclude our work in Section V.

\section{System Architecture and Application Scenario}

The converged scenario considers a cellular system in which smart UEs are under the control of the BS. In the coverage area, there also exists a group of wireless sensor nodes constructing a WSN. The smart UE gateway in the cell can provide the access for the WSN nodes, and all gateways are dual-mode and have WSN and cellular networks interfaces. Then, the data from WSN can be directly forwarded to the BS by the smart mobile gateway. The mobile UE gateway can also acquire the necessary information with the downlink data transmission from the BS or from the other fixed/mobile gateway, which is shown in Figure 1.

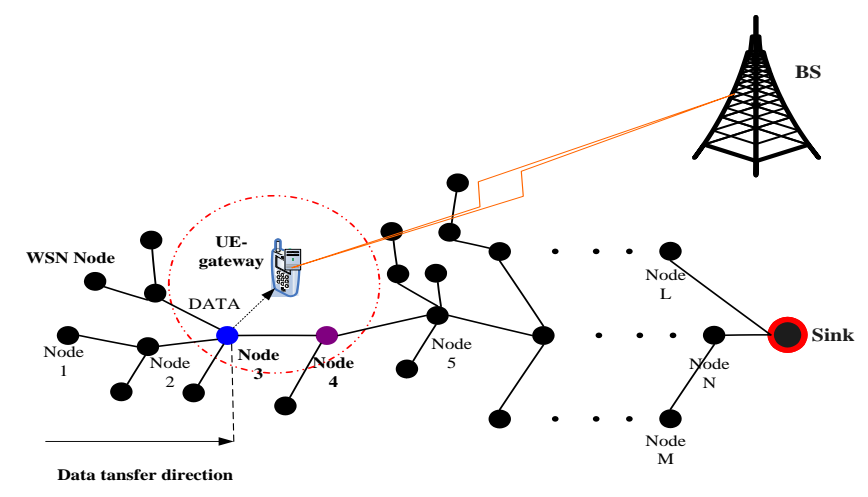

Figure 1. System Network Architecture 
In the traditional WSN and MCN networks for IoT application, the detected data from sensor nodes can be transmitted to the BS by the gateway, which usually switch the WSN data packet format to MCN data packet format according to the different network protocol stacks [15]. In this case, the switching method is implemented to change data packet format, i.e. packet header, frame control part etc. In the integrated network, a data channel between two protocol stacks is implemented in the gateway, as shown in left part of Figure. 2. The data channels between the two independent stacks are implemented to exchange packet information.

In the new converged WSN and MCN networks, the two protocol stacks are really converged where the data and signaling are shared between the network stacks [16]. Since the network architecture and air-interface are highly converged for WSN and $\mathrm{MCN}$ in the smart mobile gateway, the protocol and control signaling should also be tightly converged for a real convergence of WSN and MCN. In such a converged network, MAC and network layer protocols in the two stacks should be jointly optimized in the smart mobile gateway, which can achieve some performance gains for WSN and extend the applications of MCN. As shown in right part of Figure 2, the two protocol stacks are not independent. The data and algorithms are shared between the two stacks [17].

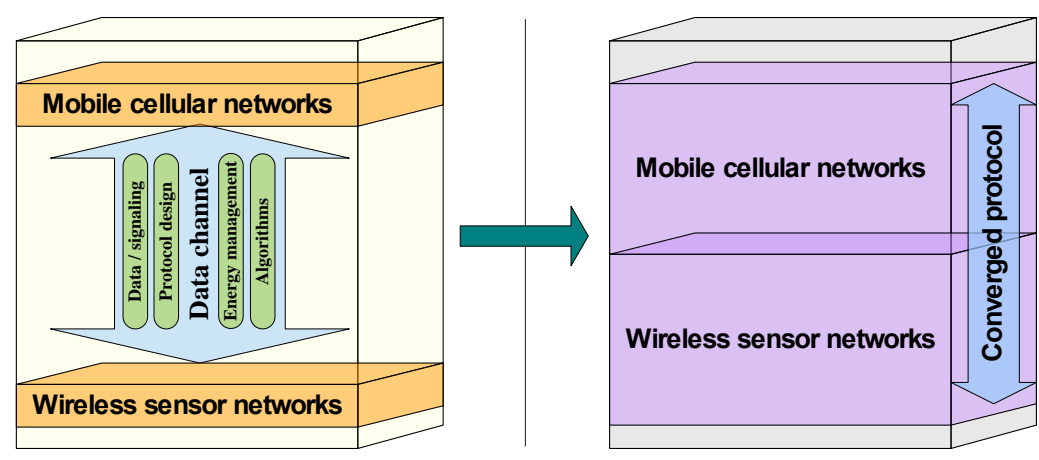

Figure 2. Converged Protocol for MCN and WSN

The application scenario for smart mobile gateway is divided into three types:

UC1: overhear the WSN data and forward to BS;

When the smart mobile gateway entries a WSN area, it begins to overhear the downlink control signaling and data, if it hears the control signaling. After receiving the broadcast message from sensor node, the UE-gateway does not need to execute CSMA$\mathrm{CA}$ and is just use the channel preemptively, which will access the channel with the highest priority without any back-off period (non-CSMA). And the sensor nodes can transmit the detected data to BS through the gateway. After some time, UE maybe leave this zone, it causes the mobile gateway selection/reselection.

UC2: act as gateway for WSN (replacement/reselection or load balance);

After a UE acting as new mobile UE gateway enters the coverage of the WSN, it may cause the gateway (re)selection or even regrouping of the wireless sensor nodes. How to make a balanced trade-off between the performance gain and energy consumption via a robust (re)selection and grouping algorithms is an essential issue. Research the gateway (re)selection algorithms and wireless sensor nodes grouping algorithms when certain UE gateways are arriving at the coverage of the WSN will become the other important part. Besides, we will design load-balancing mechanism among different UE gateways and adjust the connected number of wireless sensor nodes in order to avoid the transmission congestion and decrease the energy unbalance.

UC3: UE activates/deactivates WSN;

During the moving process of mobile smart gateway, it may enter/exit a WSN blind area. In fact, the BS doesn't know the exact position of these WSN nodes but know the 
general position of these WSN nodes. After UE entries the blind zone, BS will send downlink control signaling to SMART MOBILE GATEWAY and make SMART MOBILE GATEWAY broadcast activate message. Then the WSN nodes will choose the optimal path to form the network. After the network is formed, SMART MOBILE GATEWAY sends the information and detected data of the WSN nodes to BS. After collecting enough information, it moves to the next position and re-forms the topology of network.

\section{Service Requirements for WSN-MCN System}

In the converged WSN-MCN systems, data delivery models of smart gateway can be divided in to four types: periodical, event-driven, query-driven, continuous delivery models [18-19].

O Periodical:

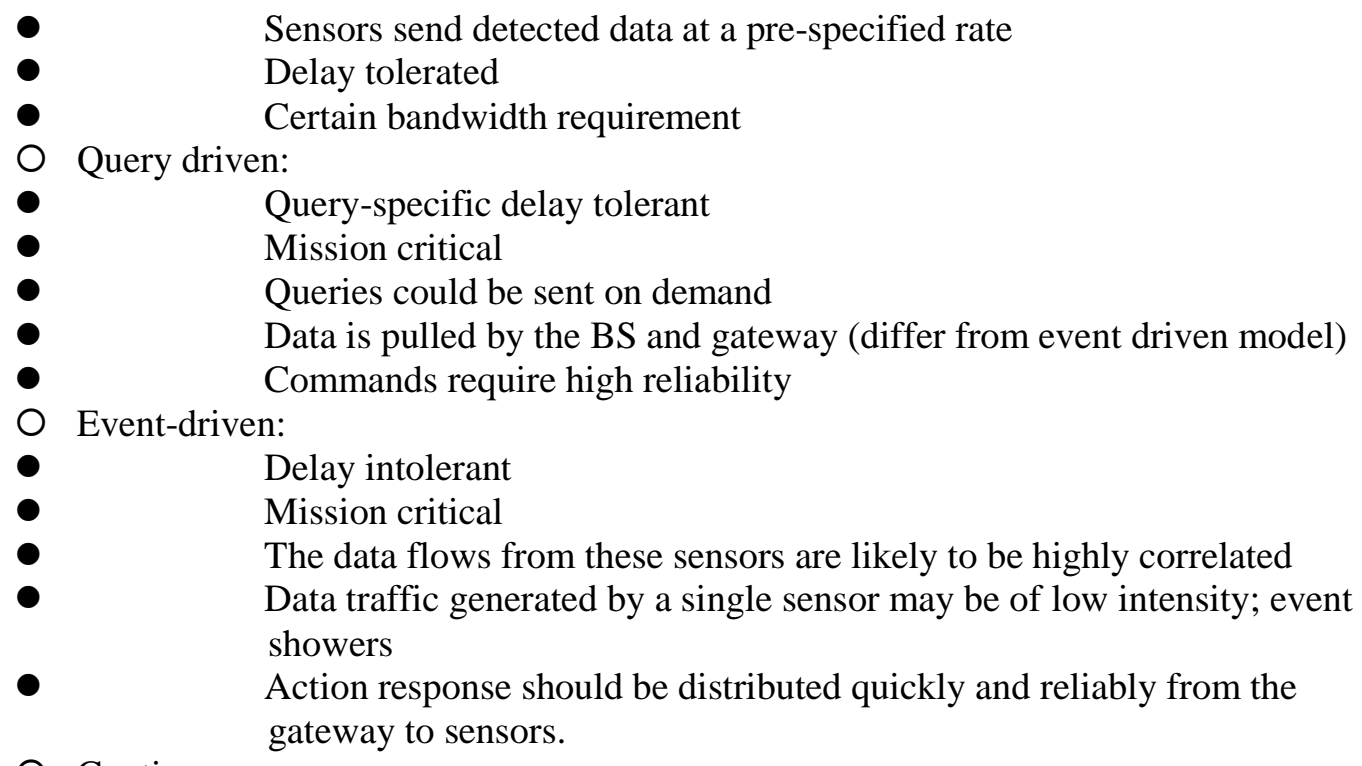
gateway to sensors.

Continuous:

Sensors send their data continuously to the data center, which is mainly about the video, image, audio or video data

Delay constrained

Certain bandwidth requirement

Packet loss can be tolerated to a certain extent

Converged WSN-MCN system shall provide QoS measures for the services provided by mobile gateway, which is shown in Table 1. Applications have different QoS requirements, such as data accuracy, reliability, latency, etc [20-22]. 
Table 1. Service Class Types

\begin{tabular}{|c|c|c|c|c|}
\hline $\begin{array}{c}\text { Service Class } \\
\text { properties }\end{array}$ & $\begin{array}{c}\text { Service Class } \\
\text { Mobility }\end{array}$ & $\begin{array}{c}\text { Service Class } \\
\mathbf{2}\end{array}$ & $\begin{array}{c}\text { Service Class } \\
\mathbf{3}\end{array}$ & $\begin{array}{c}\text { Service Class } \\
\mathbf{4}\end{array}$ \\
\hline $\begin{array}{c}\text { Group } \\
\text { Communications }\end{array}$ & Unicast & Multicast & Unicast & Unicast \\
\hline Authentication & Yes & Yes & Yes & Yes \\
\hline Integrity & Yes & Yes & Yes & Yes \\
\hline $\begin{array}{c}\text { Scheduling Delay } \\
\text { Tolerance }\end{array}$ & Tolerant & Tolerant & Best Effort & Interactive \\
\hline Peak Data Rate & $100 \mathrm{kbps}$ & $100 \mathrm{kbps}$ & $1 \mathrm{Mbps}$ & $>1 \mathrm{Mbps}$ \\
\hline Confidentiality & Yes & Yes & Yes & Yes \\
\hline $\begin{array}{c}\text { Packet Loss } \\
\text { Tolerance }\end{array}$ & None & None & Low(None?) & High \\
\hline $\begin{array}{c}\text { Device Integrity } \\
\text { Check }\end{array}$ & Yes & No & No & No \\
\hline Persistence & No & No & No & Yes \\
\hline Confirm & Yes & No & Transaction & No \\
\hline Priority & Low & Normal & High & Low \\
\hline
\end{tabular}

As WSN-MCN applications and services are supported over the existing network infrastructure, the emergency data are often carried over the network infrastructure to provide alarm notification. Besides the prioritization of certain types of data, efficient traffic and resource management for the sensed data may increase the QoS of WSN applications and services, as in general the service application transaction is usually very high. The following WSN applications and services requirements are placed on the perspective of the market they serve, which is shown in Table 2 [23-26].

Table 2. Service Application Requirements

\begin{tabular}{|l|l|}
\hline \multicolumn{1}{|c|}{ Service applications } & \multicolumn{1}{c|}{ Requirements } \\
\hline $\begin{array}{l}\text { e-Health care: } \\
\text { Monitoring vital signs } \\
\text { Web Access Telemedicine points } \\
\text { Remote diagnostics }\end{array}$ & $\begin{array}{l}\text { Need to divide the transmission service types, } \\
\text { emergency communication should have a high } \\
\text { priority. } \\
\text { Guarantee different service having different } \\
\text { delay and packet loss level. } \\
\text { Manage the mass concurrent data transmission } \\
\text { takes place in some applications. } \\
\text { Avoid access concentration to a single channel } \\
\text { resource. }\end{array}$ \\
\hline
\end{tabular}




\begin{tabular}{|c|c|}
\hline $\begin{array}{l}\text { Metering: } \\
\text { Power } \\
\text { Gas } \\
\text { Water } \\
\text { Heating } \\
\text { Grid control } \\
\text { Industrial metering }\end{array}$ & $\begin{array}{l}\text { Monitored and controlled by a centralized } \\
\text { entity outside or inside the network operator } \\
\text { system. } \\
\text { The centralized entity will inform or poll the } \\
\text { metering device when it needs measurement } \\
\text { information. } \\
\text { The network should be optimized to enable a } \\
\text { mass of sensor in a particular area to transmit } \\
\text { data almost simultaneously. } \\
\text { For the mobile UE can move, the sensor } \\
\text { module should be able to communicate with } \\
\text { mobility. } \\
\text { It should have the ability to perform secure } \\
\text { transactions between the sensor module and the } \\
\text { controlled metering device } e . g \text {. stopping the } \\
\text { delivery. } \\
\text { An object may be able to communicate in a } \\
\text { peer-to-peer manner with any other connected } \\
\text { object. In this case, packet flows should be } \\
\text { supported between these objects. } \\
\text { The system should support mutual } \\
\text { authentication and authorization between the end- } \\
\text { user and the application or capability in the } \\
\text { service layer. }\end{array}$ \\
\hline $\begin{array}{l}\text { Remote Monitoring/Control: } \\
\text { Environments surveillance } \\
\text { Pumps surveillance } \\
\text { Pollution and disaster surveillance }\end{array}$ & $\begin{array}{l}\text { Located in remote areas and ideally are } \\
\text { untouched after installation for many years. } \\
\text { The real time at which communication takes } \\
\text { place is less important, but low communication } \\
\text { costs are extremely important. } \\
\text { Allowing communication to take place during } \\
\text { low traffic time period } \\
\text { Dynamically adjust these time periods based on } \\
\text { the actual network traffic load at a specific time. } \\
\text { Application should be able to request the same } \\
\text { report in an on-demand mode. } \\
\text { The system should support the capability to } \\
\text { remotely change the working state of a sensor } \\
\text { e.g. enable or disable. }\end{array}$ \\
\hline $\begin{array}{l}\text { Security: } \\
\text { Surveillance systems } \\
\text { Control of physical access (e.g. to } \\
\text { buildings) } \\
\text { Theft /Vandalism application }\end{array}$ & $\begin{array}{l}\text { More susceptible to alarm if moving by } \\
\text { unauthorized persons. } \\
\text { Security mechanisms (The network can not } \\
\text { prevent it but can detect it as early as possible in } \\
\text { order to avoid stealing or breaking) } \\
\text { Monitoring the mobile information(If a known } \\
\text { stationary devices moves, it can be concluded } \\
\text { that the device has been stolen and thus the alarm } \\
\text { activated/deactivated) }\end{array}$ \\
\hline $\begin{array}{l}\text { Tracking \& Tracing: } \\
\text { Fleet Management } \\
\text { Traffic information } \\
\text { Road tolling }\end{array}$ & $\begin{array}{l}\text { Allows for the possibility of preventing the } \\
\text { theft itself or recovering the vehicle, for example } \\
\text { by theft tracking, sensor module should be able to } \\
\text { get its detailed location. }\end{array}$ \\
\hline
\end{tabular}




\begin{tabular}{|l|l|}
\hline Road traffic optimisation/steering & $\begin{array}{l}\text { The sensor module should be able to get its } \\
\text { detail location so that the rescue can find it easy. } \\
\text { For the entity moves fast, the sensor module } \\
\text { should be able to communicate in a mobility } \\
\text { mode. } \\
\text { The sensor module and its interfaces are able to } \\
\text { survive and operate after a shock caused by an } \\
\text { accident. } \\
\text { The communication should have a high priority } \\
\text { when it indicates an accident happens. }\end{array}$ \\
\hline
\end{tabular}

\section{Technical Challenges for Smart Mobile Gateway}

\subsection{QoS-guarantee Architecture}

The conventional scheme to guarantee the WSN transmission QoS mainly focus on the routing schemes improvement, i.e., SAR (sequential assignment routing), SPEED (A Stateless Protocol for Real-Time Communication), ReInForM (Reliable Information Forwarding using Multiple Paths in Sensor Networks), ESRT (Event to Sink Reliable Transport). The converged WSN-MCN can make QoS support more challenging to improve the WSN energy efficiency by using the smart mobile gateway [27]. Besides multiple improved routing schemes, certain efficient traffic control and resource management can also guarantee WSN QoS with the help of MCN and gateway [28-29].

In MAC layer, the smart mobile gateway needs to convey the efficient control information to/from the BS for convergence optimization. The gateway also needs to manage the subordinate WSN nodes, i.e. request the resources from BS and allocate the resource to WSN nodes for data transmission. Furthermore, the smart mobile gateway can map the WSN data and resource requests to MCN frame with the WSN service-type, and reports to the BS. And then, the BS allocates different WSN channel group to decrease the interference and guarantee the transmission quality of service $(\mathrm{QoS})$. The converged networks make QoS more challenging to improve the WSN efficiency.

In network layer, WSN topology can be reported to BS periodically. When a mobile gateway enters the coverage area of WSN, it may cause the re-clustering of sensor nodes and network topology changing. How to achieve a performance gain during the WSN re-clustering process is an essential issue under the help of smart mobile gateway and MCN. As the BS acting as database server, the further research of routing table updating and sharing is also necessary during the MCN and WSN convergence.

\subsection{Access Gateway Selection/Re-selection}

In the large coverage area of BS, there are also many sensor nodes constructing WSN. The mobile gateway can provide access for the WSN nodes. After a smart UE acting as mobile gateway and entering the coverage of WSN, it may cause the gateway reselection or even regrouping for sensor nodes [30]. In the conventional WSN, when the mobile UE gateway moves into the coverage area of the sensor nodes, they broadcast POLL packets to the sensor nodes, and provide the access for them [31-33]. However, when the serving gateway is leaving its responsible area, it will not tell the sensor nodes about its leaving. In this scenario, gateway leaving will cause WSN topology of this area reconstruct, which will bring out overhead of the signaling between mobile UE gateway and WSN nodes.

In the converged architecture, how to make a tradeoff between the performance gain and signaling/computing design in the gateway re-selection and sensor nodes regrouping process is an essential issue for WSN. In the process of UE gateway re- 
selection, mobile gateway level can be defined as an integrated index of UE gateway's status, i.e. mobility state, probability of leaving, capacity availability, and channel quality etc.

\subsection{Load Balancing for WSN}

There may be many UEs which play the role of mobile gateways for WSN. Load unbalance will often appear which one UE gateway has more sensor nodes (high traffic) and its neighbor UE gateway has fewer nodes (lower traffic). And this will cause faster energy consumption of some sensor nodes. In the traditional algorithm, the load balance is achieved when the network is initiated or the new sensor node joins to a cluster of WSN [34-35]. In the entering process, the new sensor node broadcasts access-request, and then the sink node which received the request will send a reject-ACK or acceptACK to the sensor node based on its load information.

In the converged WSN-MCN system, the mobile UE gateways should report their load information to BS and BS will manage/control the load balance of UE gateways. In the load balance process, load balancing capability should be designed for how many nodes and their services can be balanced to neighbor UE gateway. Energy efficiency is another major concern in the design of WSN's load balance, where dynamic adaptive routing protocol should be designed for data packets optimal transmission.

\subsection{Tradeoff between Energy-efficiency and Complexity}

The WSN may improve the energy efficiency based on the help of MCN and achieve better system performance. For instance, the power consumption can be reduced greatly in the gateway re-selection and load balance process for WSN, but the WSN complexity is increased with the additional signaling design for neighbor mobile gateways scanning. Therefore, there are still several important issues for balancing energy-efficiency and algorithm complexity which still require further research [36]. We detail several potential points as follows:

Software challenge of mobile gateway: Both MCN and WSN have different working capacity, resource management requirements. This heterogeneity makes the design of converged system architecture difficult, especially for the mobile gateway. The software for gateway should have the ability to analyze the collected data, overhear signaling and can refine the useful information. It should add some improved scheduling algorithm for various WSN services in order to achieve the joint resource allocation to guarantee the multi-types services. The UE gateway should construct the resource mapping table in the networks convergence process which will help the network to use channel resource effectively and improve energy efficiency. At last, it needs to transmit the highest priority information and control signaling to the WSN or MCN with the minimum delay.

Hardware challenge of mobile gateway: Normal gateway including WSN and MCN interfaces maybe used in extremely difficult environment. Moreover, the mobile gateways maybe move in patrolling mode application. So there are two issues should be considered to address the hardware challenge. One is packaging, and we need to make the gateway water-proof, fire-proof. The other is we need to improve the radio module and make it more precise to support the application in moving scenario.

\section{Conclusions}

MCN and WSN are evolving from heterogeneous to converged in order to satisfy the increasing requirements of IoT communications, where many joint optimized methods and technical challenges still exist. In this survey, we analyze the system architecture for WSN-MCN converged networks based on the smart mobile gateway. Further, we investigate system application requirement for IoT 
QoS guarantee in the WSN data transmission process. Finally, we discuss the key technical challenges and research points based on mobile gateway with the unique characteristics of converged MCN and WSN. We are convinced that the mobile gateway in the MCN and WSN converged process should widely be researched in order to stimulate M2M application in the future, which can also promote the industry development of the Internet of things (IoT).

\section{Acknowledgments}

This work was supported by the National Natural Science Foundation of China (No.61302113), Shanghai Natural Science Foundation (No.13ZR1440800), Shanghai Rising-Star Program (14QB1404400), Shanghai Science and Technology Committee Program (14DZ2294500) and Shanghai Key Laboratory of Specialty Fiber Optics and Optical Access Networks (SKLSFO2014-03).

\section{References}

[1] J. Zheng and A. Jamalipour, "Wireless Sensor Networks: A Networking Perspective," 1st end, Wiley Press, New York, (2009), pp. 125-138.

[2] S. Bouckaert and E. D. Poorter, "Strategies and challenges for interconnecting wireless mesh and wireless sensor networks", Wireless Personal Communications, vol. 3, no. 53, (2010).

[3] Z. J. Li, M. Li and J. L. Wang, "Ubiquitous data collection for mobile users in wireless sensor networks", Proceedings of the IEEE INFOCOM, (2011).

[4] H. Zara and F. B. Hussain, "QoS in wireless multimedia sensor networks: a layered and cross-layered approach”, Wireless Personal Communications, vol. 1, no. 75, (2014).

[5] L. He, J. P. Pan and J. D. Xu, "A progressive approach to reducing data collection latency in wireless sensor networks with mobile elements", IEEE Trans on Mobile Computing, vol. 7, no. 12, (2013).

[6] S. Basagni, A. Carosi, E. Melachrinoudis, C, Petrioli and Z. M. Wang, "Controlled sink mobility for prolonging wireless sensor networks lifetime”, Wireless Networks, vol. 6, no. 14, (2008).

[7] G. L. Xing, T. Wang and W. J. Jia, "Rendezvous design algorithms for wireless sensor networks with a mobile base station", Proceedings of the ACM MobiHoc, (2008)

[8] S. Gao, H. Zhang and S. K. Das, "Efficient data collection in wireless sensor networks with pathconstrained mobile sinks", IEEE Trans. on Mobile Computing, vol. 5, no. 10, (2011).

[9] G. Anastasi, E. Borgia and M. Conti, "A hybrid adaptive protocol for reliable data delivery in WSNs with multiple mobile sinks", The Computer Journal, vol. 2, no. 54, (2011).

[10] W. F. Liang, J. Luo and X. Xu, "Prolonging network lifetime via a controlled mobile sink in wireless sensor networks", Proceedings of the IEEE GlobeCom, (2010).

[11] J. Zhang, L. H. Shan and H. L. Hu, "Mobile cellular networks and wireless sensor networks: toward convergence", IEEE Communications Magazine, vol. 3, no. 50, (2012).

[12] G. V. Crosby and F. Vafa, "Wireless sensor networks and LTE-A network convergence", Proceedings of the 38th Annual Conference on Local Computer Networks, (2013).

[13] M. Marta and M. Cardei, "Using sink mobility to increase wireless sensor networks lifetime", Proceedings of International Symposium on a World of Wireless, Mobile and Multimedia Networks, (2008)

[14] X. Xu, J. Luo and Q. Zhang, "Delay tolerant event collection in sensor networks with mobile sink", Proceedings of the IEEE Infocom, (2010).

[15] D. Puccinelli and M. Haenggi, "Wireless sensor networks: applications and challenges of ubiquitous sensing", IEEE Circuits and Systems Magazine, vol. 3, no. 5, (2005).

[16] M. Gatzianas and L. Georgiadis, "A distributed algorithm for maximum lifetime routing in sensor networks with mobile sink", IEEE Transaction on Wireless Communications, vol. 3, no. 7, (2008).

[17] L. H. Shan, Z. H. Li and H. L. Hu, "Converged Mobile Cellular Networks and Wireless Sensor Networks for Machine-to-Machine Communications", KSII Transactions on Internet and Information Systems, vol. 1, no. 6, (2012).

[18] S. A. A. Omari and P. Sumari, "An overview of mobile ad hoc networks for the existing protocols and applications", International Journals on applications of graph theory in wireless and ad hoc networks and sensor networks, vol. 1, no. 2, (2010).

[19] H. Zara and F. B. Hussain, "QoS in Wireless Multimedia Sensor Networks: A Layered and CrossLayered Approach", Wireless Personal Communications, vol. 1, no. 75, (2014).

[20] J. Wang, X. Q. Yang, Z. Q. Zhang, B. Li and J. U. Kim, "A Survey about Routing Protocols with Mobile Sink for Wireless Sensor Network", International Journal of Future Generation Communication and Networking, vol. 7, no. 5, (2014). 
[21] Z. Y. Chen, G. Yang, L. Chen and J. Wang, "An Algorithm for Data Aggregation Scheduling with Long-lifetime and Low-latency in Wireless Sensor Networks", International Journal of Future Generation Communication and Networking, vol. 5, no. 4, (2012).

[22] ITU-T Y.2012, Recommendation ITU-T Y.2012. Functional requirements and architecture of the NGN (Release 1). (2006)

[23] http://portal.etsi.org/portal/server.pt/community/M2M/319

[24] http://www.itu.int/itu-t/recommendations/rec.aspx?id=10235

[25] http://www.3gpp. org/ftp/Specs/html-info/22368.htm

[26] http://www.3gpp. org/ftp/Specs/html-info/23868.htm

[27] G. V. Crosby and F. Vafa, "Wireless Sensor Networks and LTE-A Network Convergence", Proceedings of the 38th Annual Conference on Local Computer Networks, (2013).

[28] T. Melodia and I. F. Akyildiz, "Cross-layer QoS-aware communication for ultra wide band wireless multimedia sensor networks", IEEE Journal on Selected Areas in Communications, vol. 5, no. 28, (2010).

[29] "Effective M2M gateway selection algorithms for geographical region-based query", Proceedings of International Conference on Information and Communication Technology Convergence, (2010).

[30] D. Bae, W. Choi, J. Kwon and H. Choo, "A novel opportunistic greedy forwarding scheme in wireless sensor networks", KSII Transactions on Internet and Information Systems, vol. 5, no. 4, (2010).

[31] S. A. A. Omari and P. Sumari, "An overview of mobile ad hoc networks for the existing protocols and applications", International Journals on applications of graph theory in wireless ad hoc networks and sensor networks, vol. 2, no. 1, (2010).

[32] H. Abusaimeh and S. H. Yang, "Dynamic cluster head for lifetime efficiency in WSN", International Journal of automation and computing, vol. 6, no. 1, (2009).

[33] M. Gatzianas and L. Georgiadis, "A distributed algorithm for maximum lifetime routing in sensor networks with mobile sink", IEEE Trans. on Wireless Communications, vol. 7, no. 3, (2008)

[34] M. Masdari and M. Tanabi, "Multipath Routing protocols in Wireless Sensor Networks: A Survey and Analysis", International Journal of Future Generation Communication and Networking, vol. 6, no. 6, (2013).

[35] S. Bouckaert, E. D. Poorter and B. Latré, "Strategies and challenges for interconnecting wireless mesh and wireless sensor networks", Journcal of Wireless Personal Communications, vol. 3, no. 53, (2010).

[36] B. An, T. T. Duy and H. Kong, "A cooperative transmission strategy using entropy-based relay selection in mobile ad-hoc wireless sensor networks with rayleigh fading environments", KSII Transactions on Internet and Information Systems, vol. 2, no. 3, (2009).

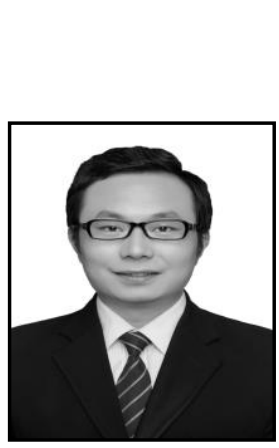

\section{Authors}

Lianhai Shan, he received his BSc and MSc in Electrical Engineering and Automation from Wuhan University of Technology, Wuhan, China in 2003 and 2006. He received the $\mathrm{PhD}$ degree in communication engineering from Tongji University, Shanghai, China in 2009. In 2008, he carried out the research in National Institute of Informatics and University of Essex as a visiting student. His research interests include QoS and MAC for heterogeneous wireless networks.

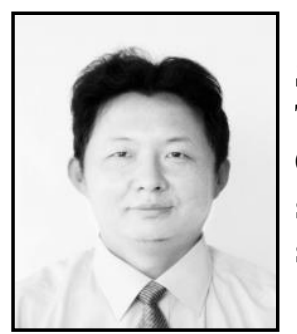

Weidong Fang, he is a Ph.D. and an associate professor in Shanghai Institute of Microsystem and Information Technology (SIMIT), Chinese Academy of Sciences, Shanghai, China. Currently, his research interests are information security in wireless sensor work, including trust management, secure network coding and secure routing protocol. 


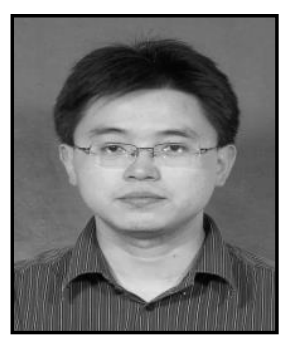

Qiu Yunzhou, he received the B.S. degree and M.S. degree from the School of Manufacturing Science and Engineering of Sichuan University in 2001 and 2004, and Ph.D. degree from the Communication and Information System of Shanghai Institute of Microsystem and Information Technology (SIMIT), CAS in 2007. $\mathrm{He}$ is currently associate professor of SIMIT. His research interests include QoS guarantee and resource allocation for wireless sensor networks.

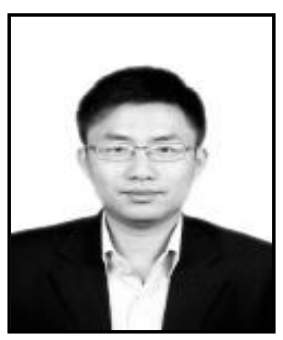

Wei He, he received his Ph.D. Degree from the Communication and Information System of Shanghai Institute of Microsystem and Information Technology (SIMIT), CAS in 2013. Now he works as associate professor in SIMIT, CAS. His research interests include the sound vibration sensor array signal processing, target tracking and locating.

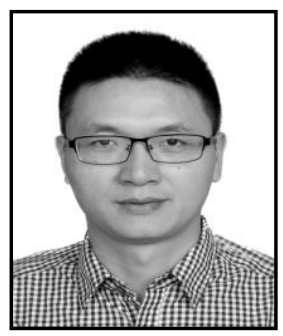

Yanzan Sun, he received his BSc and MSc in Electrical Engineering and Automation from Qingdao University of Technology, China in 2004 and 2007. He received the $\mathrm{PhD}$ degree in communication engineering from Tongji University, Shanghai, China in 2011. In 2009, he carried out the visiting research in Columbia University as a visiting student. His research interests include cognitive radio and resource allocation for mobile cellular networks. 
International Journal of Future Generation Communication and Networking Vol. 9, No.9, (2016) 